accounts for the increase in laser output on the short wavelength side as more power is coupled out of the cavity. The tuning curve shows small but sharp fluctuations with wavelength which we believe to be caused by stress induced birefringence in the doped fibre. These in turn cause the polarisation state to rotate with the wavelength giving varying degrees of loss from the birefringent tuning element. ${ }^{12}$ In the future this may be overcome by introducing small but controlled amounts of birefringence in the active fibre. ${ }^{12}$

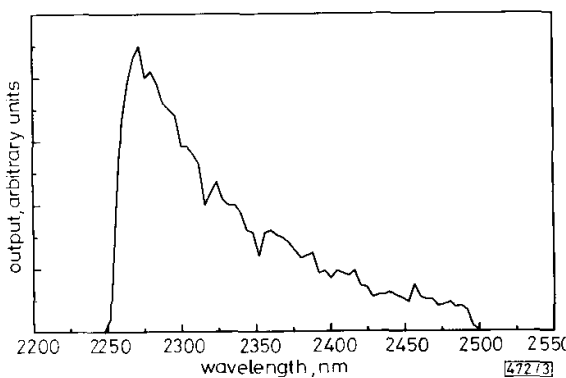

Fig. 3 Observed tuning range for thulium doped fibre laser on $2.31 \mathrm{\mu m}$ laser transition

Conclusion: We have reported preliminary results on laser action in a thulium doped monomode fluoride fibre over the tuning range from 2.25 to $2.5 \mu \mathrm{m}$. At this latter wavelength such a source becomes a promising candidate for application as a transmitter for the future ultralow loss transmission systems using fluoride based fibres. Our results are, as yet, unoptimised with considerable scope for further improvement. In particular we believe that modest increases in the fibre NA will lead to reductions in the threshold pump power coupled with increased output power at $2.5 \mu \mathrm{m}$. Future studies will also address the possible application of this medium as an inline amplifier, especially targeted at the submarine systems arena.

R. M. PERCIVAL
S. F. CARTER
D. SZEBESTA
S. T. DAVEY
W. A. STALLARD
BT Laboratories, Martlesham Heath
Ipswich, Suffolk, IP5 7 RE
United Kingdom

Ist August 1991

References

1 CARTER, S. F., MOORE, M. W., SZEBESTA, D., WILLIAMS, J. R., RANSON, D., and FRANCE, P. W.: 'Low loss fluoride fibre by reduced pressure casting', Electron. Lett., 1990, 26, pp. 2115-2117

2 eWING, K. J., JaGanathan, J., ROURKE, R. M., and AGgarWaL, I. D.: 'Purification and analysis of fluoride raw materials at sub $\mathrm{ppb}$ levels'. Proc. 5th Halide Glass Symp. Japan, 1988, pp. 139-145

3 GARNHAM, R. A., LEARMOUTH, M. D., RIMINGTON, J. J., ALI, A. S. M., ROBERTSON, M. J., and STALLARD, W. A.: ' $140 \mathrm{Mbit} / \mathrm{s}$ receiver perROBERTSON, M. J., and STALLARD, W. A.: '140 Mbit/s receiver performance at $2.4 \mu \mathrm{m}$

24, pp. 1416-1417

4 martinelit, R. U., and Zamerowski, T. J.: 'InGaAs/InAsPSb diode lasers with output wavelengths at $2.52 \mu \mathrm{m}$ ', Appl. Phys. Lett., 1990, 56, pp. 125-127

5 AKIBA, S., MaTSUSHIMA, Y., IKETANI, T., and USAMl, M.: 'InAsPSb/ InAs diode laser emitting at $2.5 \mu \mathrm{m}$ range', Electron. Lett., 1988, 24, pp. 1069-1071

6 RONARC'H, D, GUIBERT, M AUZFL, F MFCHENIN, D., ALLAIN, J. Y., and POIGNANT, H.: '35 dB optical gain at $2.716 \mu \mathrm{m}$ in erbium doped ZBLAN fibre pumped at $0.642 \mu \mathrm{m}$, Electron. Lett., 1991, 27, pp. $511-513$

7 FRANCE, P. W., Carter, S. F., MOORE, M. W., and Day, C. R.: 'Progress in fluoride fibres for optical communications', Br. Telecom. Technol. J., 1987, 5, pp. 2844

8 ALLAIN, J. Y.. MONERIE, M., and POIGNANT, H.: 'Energy transfer in $\mathrm{Er}^{3+} / \mathrm{Pr}^{3+}$-doped fluoride glass fibres and application to lasing at 2.7 m', Electron. Lett., 1991, 27, pp. 445-447

9 AlLAiN, J. Y., MONERIE, M., and POIGNANT, H.: 'Tunable CW lasing around $0.82,1.48,1.88$, and $2.35 \mu \mathrm{m}$ in thulium doped fluorozirconate fibre', Electron. Lett., 1989, 25, pp. 1660-1662
10 GUERY, C., ADAM, J. L., and LUCAS, J.: 'Optical properties of $\mathrm{Tm}^{3+}$ ions in indium-based fluoride glasses', $J$. Luminescence, 1988, 42, pp. $181-189$

11 SMART, R. G., CARTER, J. N., TROPPER, A. C., and HANNA, D. C.: 'Continuous-wave oscillation of $\mathrm{Tm}^{3+}$ - A.ped fuorozirconate fibre Continuous-wave oscillation of Tm 3 t 23 . lasers around $147 \mu \mathrm{m}, 1.9 \mu \mathrm{m}$ and $23 \mu \mathrm{m}$ $790 \mathrm{~nm}$, Opt. Commun. 1991, 82, pp. 563-570

12 hANNA, D. C., PERCIVAL, R. M., PERRY, I. R., SMART, R. G., SUNI, P. J., and TROPPER, A. C.: 'An ytterbium-doped monomode fibre laser: broadly tunable operation from $1.010 \mu \mathrm{m}$ to $1.162 \mu \mathrm{m}$ and three level operation at $974 \mathrm{~nm}, J$. Mod. Opt., 1990, 37, pp. 517-525

\section{SWITCHED-CAPACITOR BROADBAND NOISE GENERATOR FOR CMOS VLSI}

Indexing terms: Oscillators, Switched-capacitor circuits, Circuit design, Integrated circuits

A switched-capacitor circuit is reported for the generation of broadband white noise in MOS VLSI. It is based on the implementation of a very simple chaotic discrete-time system. The concept is demonstrated via a $3 \mu \mathrm{m}$ CMOS double-metal double-poly monolithic prototype yielding a $4 \mathrm{~V}$ peak-topeak signal with a flat power density spectrum from $D C$ to about half the clock frequency.

Introduction: Noise sources are basic building blocks for instrumentation and communication systems. ${ }^{1}$ In particular, white noise generators are used in speech-processing problems ${ }^{2}$ and for testing the dynamic behaviour of many electronic systems. ${ }^{3}$ They can also be used for the generation of secure nonrepeatable pseudorandom number sequences. ${ }^{4} \mathrm{~A}$ more novel application of noise sources is in VLSI neura networks incorporating stochastic learning techniques. ${ }^{5}$ Cascading a white noise source and a filter is a common approach for obtaining coloured noise. ${ }^{6}$ In all of these applications, implementing on-chip noise sources with reasonable area occupation is an ideal target.

A common technique for generating analogue noise signals is to exploit some natural noise source, for instance a Zener diode ${ }^{7}$ This approach has two drawbacks for VLSI. First, very low power signals are obtained, imposing the need for using large gain amplifiers. It may produce spurious correlation in cases where multiple sources are required. ${ }^{5}$ Secondly, appropriate sources may not be well suited for on-chip implementation in a given technology, thereby forcing the designer to consider off-chip components. The technique in this Letter overcomes both drawbacks by exploiting the random properties of deterministic chaos. ${ }^{8}$ A very simple switched-capacitor white noise generator is presented which is well suited for CMOS, BiCMOS and GaAs technologies, and is demonstrated via a CMOS prototype. The circuit is simpler and smaller than digital noise generators composed by feedback shift registers and lowpass filters, ${ }^{7}$ also exhibiting, as compared to the latter generators, the feature of providing fully aperiodic signals.

Circuit description: The proposed circuit is intended to implement the following nonlinear discrete-time system

$$
x(n)=\left\{\begin{aligned}
A+B x(n-1) & \text { for } x(n-1)<0 \\
-A+B x(n-1) & \text { for } x(n-1)>0
\end{aligned}\right.
$$$$
n=1,2,3 \text {. }
$$

where $A$ and $B$ are real parameters and $n$ symbolises the iteration index. This model is a member of the generic class of discrete maps which are recognised to be the simplest mathematical models able to generate chaotic (i.e. random-like) signals. ${ }^{8}$

The implementation of eqn. 1 requires only linear amplification, summation and comparison and is hence suitable for SC circuits. Fig. 1 shows an offset-free parasitic-insensitive SC schematic diagram for eqn. 1. Notice that just two opamps

ELECTRONICS LETTERS 10th October 1991 Vol. 27 No. 21 
and one comparator are required. Opamp $\mathrm{OA} 1$ and related capacitors perform the weighted summation required in eqn. 1 , whereas opamp OA2 is used to implement a delay. The nonlinearity is realised via a phase-reverser switch arrangement controlled by the comparator. Depending on the value of $Z$, this arrangement means $E_{x E F}$ is to be either added or subtracted at the output of the opamp OA1 thereby yielding the required change of sign in eqn. 1 .
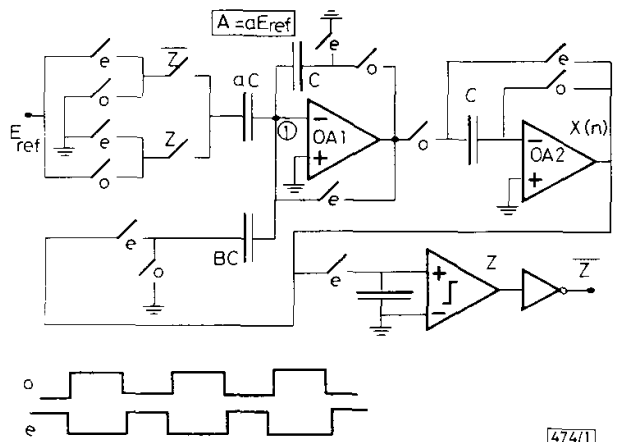

47411

Fig. 1 Parasitic-insensitive SC white noise generator

The model operation is controlled by two parameters, $A$ and $B$, whose values can be set via two capacitor ratios in Fig. 1. Parameter $B$ determines the dynamic properties of the generated signal. For $B$ less than 1 , the signal $x(n)$ converges, for each value of $A$, towards a stable equilibrium point. For $B$ greater than $2, x(n)$ diverges. In Fig. 1 this will lead the opamps into saturation. Finally, for $B$ between 1 and 2 , the values of the subsequent samples of $x(n)$ are randomly distrib uted in the interval $\{-A, A\}$. This range of $B$ values is hence the one of interest for our application.

Parameter $A$ does not influence the dynamics of eqn. 1 but it acts just as a scale factor. In practical implementations, however, its value has to be properly set to avoid locking at parasitic stable points caused by the saturation of the active devices. The design criteria are shown in Fig. 2, where we have considered two different values of $A$ for the same value of $B(1<B<2)$ and have drawn the corresponding open-loop transfer characteristics of Fig. 1 including the influence of the opamp voltage saturation. In Fig. $2 a$ parasitic stable points $Q_{1}$ and $Q_{2}$ appear at the crossing points between the transfer function characteristics and the bisecting line. For this case the circuit will evolve until it is locked either at $Q_{1}$ or at $Q_{2}$, hence not yielding random signals. On the other hand, for Fig. $2 b$ no spurious equilibria appear and random signals wil actually be obtained. The following must be fulfilled for the transfer function to be as the one in Fig. $2 b$ :

$$
A<E_{s^{+}}<A /(B-1) \quad A /(1-B)<E_{s_{-}}<-A
$$

where $E_{S+}\left(E_{S_{-}}\right)$denotes the opamp positive (negative) satura tion level.

Analysis has been made by us to, first, calculate proper settings for $A$ and $B$, and, secondly, assess the circuit robustness when the component values are randomly changed around their nominal values according to typical deviations in

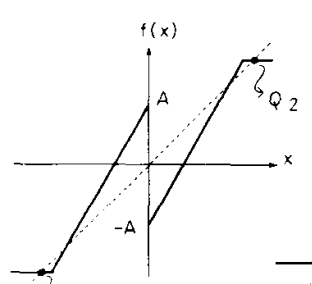

$Q$

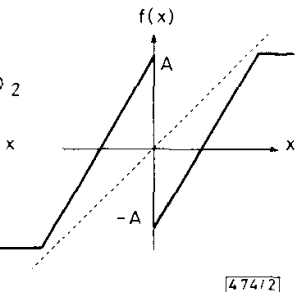

474:?
Fig. 2 Design criteria

$a$ Onset of parasitic stable points due to improper setting of $A$ $b$ Strategy to avoid parasitic stable points monolithic circuits. ${ }^{2}$ Robust stationary, regular and ergodic behaviour was observed for values of $B$ greater than $(2)^{1 / 2}$.

Experimental results: A programmable prototype of Fig. 1 has been fabricated in a $3 \mu \mathrm{m}$ CMOS $n$-well double-poly doublemetal technology. Actually a slightly modified version of Fig. 1 was considered where the value of the slope of eqn. 1 for $x<0$ (which we call $B_{1}$ ) can be controlled, independently of the corresponding value for $x>0\left(B_{2}\right)$. Two binary-weighted capacitors (with six control bits each) were used to separately adjust $B_{1}$ and $B_{2}$ over the chaotic interval, $1<\left(B_{1}, B_{2}\right)<2$. Also, a control bit was added to selectively open or close the feedback loop. All measurements reported herein are for a clock frequency of $f_{c}=200 \mathrm{kHz}$ and for a $E_{R E F}$ value yielding $A=2$. Fig. 3 corresponds to a case where the feedback loop

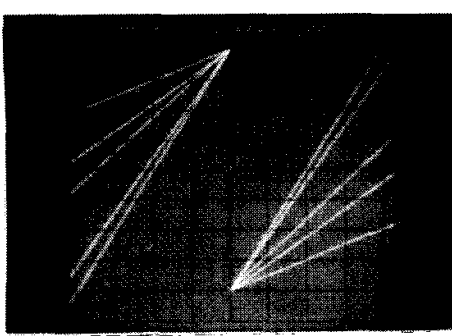

$474 / 3$

Fig. 3 Measured open-loop transfer characteristics for different $B_{1}$ and $B_{2}$ values

was opened and shows a family of open-loop transfer characteristics for different values of $B_{1}$ and $B_{2}$. Measurements in a closed loop were made for a large number of $B_{1}$ and $B_{2}$ values inside the chaotic interval. No periodicity was observed in any case. The flattest spectra were obtained for the cases where $B_{1}=B_{2}=B$. Also, the flat spectrum band was observed to decrease as the value of $B_{1}=B_{2}=B$ decreased. On the other hand, for $B_{1} \neq B_{2}$ this band not only decreased but also the generated noise became coloured. Fig. 4 illustrates the circuit operation in a closed loop. Fig. $4 a$ is for $B_{1}=B_{2}=61 / 32$, and Fig. $4 b$ is for $B_{1}=61 / 32, B_{2}=47 / 32$. For each case both the power density spectrum and a piece of the output signal (corresponding to a single oscilloscope shot) are shown. It can be seen that the spectrum in Fig. $4 a$ is flat to about $80 \mathrm{kHz}$. Actually a $1 \mathrm{~dB}$ deviation was measured from DC to $75 \mathrm{kHz}$ (35\% of the clock frequency). Also the oscilloscope pictures show how apparently coincident values of $x(n)$ result in quite different values after a few iterations thereby confirming the unpredictability of deterministic chaos.
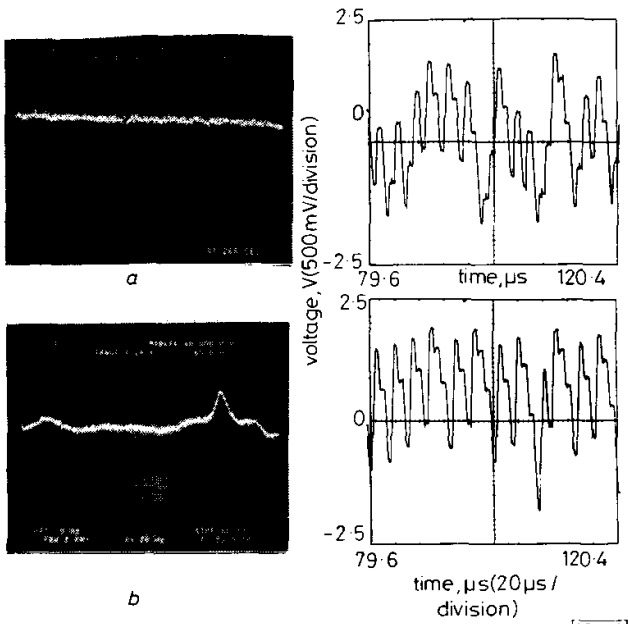

Fig. 4 Measured spectra and signals for two different $B_{1}, B_{2}$ setting a $B_{1}=B_{2}=61 / 32$

b $B_{1}=61 / 32, B_{2}=47 / 32$ 
Summarising, this Letter demonstrates that chaos, which has deserved strong academic interest in previous years, can be exploited to be used in integrated circuit engineering. The proposed generator can be used as a stand-alone IC unit or as part of a larger monolithic signal processing and computing system.

A. RODRIGUEZ-VÁZQUEZ

1st August 199

M. DELGADO

S. ESPEJO

J. L. HUERTAS

Department of Analog Design

Centro Nacional de Microelectrónica

C/Tarfia sn, 410I2-Sevilla, Spain

References

1 GUPTA, M.: 'Applications of electrical noise', Proc. IEEE, July 1975, 63, pp. $996-1010$

2 GREGORIAN, R., and TEMES, G. C.: 'Analog MOS integrated circuits for signal processing'. (John Wiley, 1986)

3 ScHOUKENS, J. et al:: 'Survey of excitation signals for FFT based signal analyzers', IEEE Trans., September 1988, IM-37, pp. 342-352

4 Bernstein, G. M., and Lieberman, M. A.: 'Secure random number generation using switched-capacitor circuits', IEEE Trans., September 1990, CAS-37, pp. 1157-1164

5 ALSPECTOR, J. et al:: 'A VLSI-efficient technique for generating multiple uncorrelated noise sources and its application to Stochastic neural networks', IEEE Trans., January 1991, CAS-38, pp $109-123$

6 CORSIN, G., and SALETTI, R.: 'A 1// power spectrum noise sequence generator', IEEE Trans., December 1988, IM-37, pp. 615-619

7 Horowitz, P., and HILL, w.: 'The art of electronics' (Cambridge University Press, 1989, 2nd edn.)

8 DEYANEY, R. L. 'An introduction to chaotic dynamical systems' (Ben./Cummings, 1986)

9 ESPEJO, S.: 'Design of analog/digital random generators'. Masters Thesis, Univ. Seville, 1990

HIGH POWER, 8.5W CW, VISIBLE LASER DIODES

Indexing terms: Semiconductor lasers, Lasers

Visible laser diodes have been fabricated from AlGaInP operating at approximately $680 \mathrm{~nm}$ to high output powers. Broad area lasers with $100 \mu \mathrm{m}$ wide emitting apertures operate to greater than $1 \mathrm{~W} \mathrm{CW}$ with a differential efficiency of $38 \%$. The threshold current densities of the material have been measured to be as low as $350 \mathrm{~A} / \mathrm{cm}^{2}$ for lasers with $30 \%$ been measured to be as low as $350 \mathrm{~A} / \mathrm{cm}^{2}$ for lasers with $30 \%$
mirror reflectivities. Monolithic bars $8 \mathrm{~mm}$ long with $50 \mu \mathrm{m}$ emitting apertures periodically spaced on $500 \mu \mathrm{m}$ centres have been fabricated which operate to $8.5 \mathrm{~W} \mathrm{CW}$.

Visible laser diodes ${ }^{1-8}$ have been under investigation for applications including optical recording and bar code scanning, both of which require nearly diffraction limited operation. Research of laser diodes that satisfy these applications has demonstrated maximum singlemode output powers of $75 \mathrm{~mW} \mathrm{CW}{ }^{1}$ Other applications which do not require singlemode operation include pumping of solid state laser material, printing, displays, and medical applications. For these applications, however, large output powers are required from the laser source. High power operation has been limited primarily by high threshold currents. The incorporation of quantum well active layers ${ }^{2}$ has significantly reduced the threshold current densities to approximately $300 \mathrm{~A} / \mathrm{cm}^{2}$. Recently, multimode gain guided laser diodes with single quantum well active regions have been operated to $350 \mathrm{~mW} \mathrm{CW}^{3}$

We discuss the operation of high power visible laser diodes that operate to output powers of $8.5 \mathrm{~W} \mathrm{CW}$ from a monolithic array of $16,50 \mu \mathrm{m}$ emitters across an $8 \mathrm{~mm}$ bar. In addition, single $100 \mu \mathrm{m}$ wide broad area diodes operate up to $1 \mathrm{~W} \mathrm{CW}$ with a differential efficiency of $38 \%$. The minimum threshold current density measured for this material is $350 \mathrm{~A} / \mathrm{cm}^{2}$.
The epitaxial structure, grown by metalorganic chemical vapour deposition (MOCVD), consists of a GaInP single quantum well active layer where the In composition is $60 \%$. The strained quantum well active region increases the gain of the active layer resulting in a lower threshold current than lattice-matched laser structures. The cladding layers are $\mathrm{Al}_{0.3} \mathrm{Ga}_{0.2} \mathrm{In}_{0.5} \mathrm{P}$. The dopants of the cladding layers are $\mathrm{Zn}$ and $\mathrm{Si}$. The cap layer is $\mathrm{GaAs}$ with an intermediate layer of GaInP. The lasers were fabricated into broad area structures by proton implantation, cleaved to cavity lengths of $500 \mu \mathrm{m}$ and bonded $p$-side down for CW operation.

Fig. 1 presents the light output as a function of input current for a $100 \mu \mathrm{m}$ wide broad area laser operating under $\mathrm{CW}$ conditions at room temperature. The cavity length of the laser is $500 \mu \mathrm{m}$ and the facet reflectivities are 4 and $95 \%$ for the front and rear facets, respectively. The maximum output is $1 \mathrm{~W} \mathrm{CW}$ with a differential efficiency of $38 \%$. The threshold current density of the laser is $600 \mathrm{~A} / \mathrm{cm}^{2}$, for lasers with no mirror coatings and for reflectivities of approximately $30 \%$, the threshold current density is $350 \mathrm{~A} / \mathrm{cm}^{2}$. The total power conversion efficiency of the laser is $27 \%$ at an output power of $200 \mathrm{~mW}$ CW.

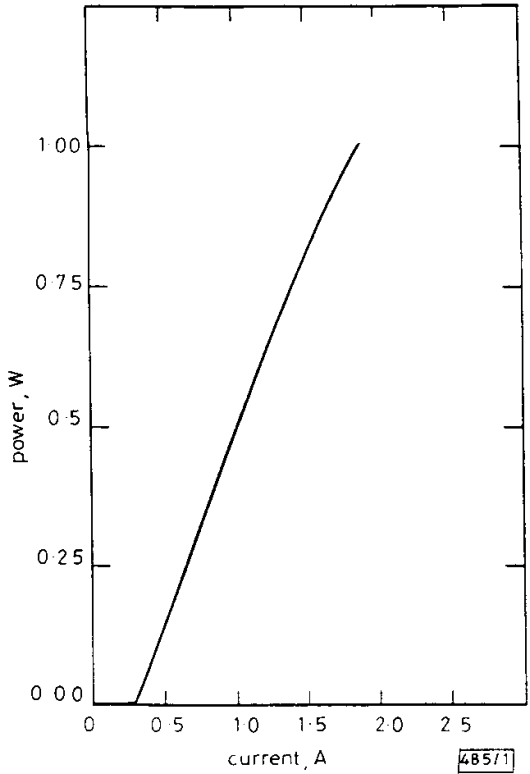

Fig. 1 Light output as function of input current for $100 \mu \mathrm{m}$ broad area laser diode

$$
P_{\text {mac }}=1 \mathrm{~W} \quad R_{f} \simeq 4 \% \quad 100 \mu \mathrm{m} \text { aperture } \quad \eta_{D}=38 \%
$$

Similar devices were fabricated at a cavity length of $1 \mathrm{~mm}$ The threshold current density changed from 600 to $350 \mathrm{~A} / \mathrm{cm}^{2}$ while the differential efficiency decreased from 38 to $22 \%$. Assuming a linear gain relationship, the extrapolated transparency current is approximately $100 \mathrm{~A} / \mathrm{cm}^{2}$, the gain coefficient is $0.013 \mathrm{~cm} \mu \mathrm{m} / \mathrm{A}$, the internal conversion efficiency is 0.95 , and the distributed loss is $53 \mathrm{~cm}^{-1}$. The values presented here are only a rough indication of the device parameters because the extrapolated data were taken from a limited number of points. However, the high distributed loss and the low gain coefficient are indicative of some of the performance limitations of visible lasers. The low gain coeflicient can be correlated to the low $T_{0}$ value of $60 \mathrm{~K}$, which is believed to be charge leakage into the separate confinement regions. The source of the high distributed loss is unknown.

Higher power monolithic sources have been fabricated by periodically spacing emitters along an $8 \mathrm{~mm}$ bar. Each aperture size is $50 \mu \mathrm{m}$ and is spaced on $500 \mu \mathrm{m}$ centres. The cavity length of the laser is $500 \mu \mathrm{m}$ and the facet coatings are 4 and $95 \%$ on the front and rear facets, respectively. Fig. 2 presents the light output as a function of the input current for the laser bar. The maximum output power is $8.5 \mathrm{~W} \mathrm{CW}$ where the 